\title{
Color Based Image Retrieval by Combining Various Features
}

\section{Bably Dolly, Deepa Raj}

\begin{abstract}
Content based image retrieval system retrieve the images according to the strong feature related to desire as color, texture and shape of an image. Although visual features cannot be completely determined by semantic features, but still semantic features can be integrate easily into mathematical formulas. This paper is focused on retrieval of images within a large image collection, based on color projection by applying segmentation and quantification on different color models and compared for good result. This method is applied on different categories of image set and evaluated its retrieval rate in different models.

Keywords: segmentation, image retrieval, color feature
\end{abstract}

\section{INTRODUCTION}

In today's scenario, due to utilization of internet and web technologies there is wide spread range of collection of databases with enormous categories of images. Different type of information are going to be gathered from online in terms of categorized books, learning content, newspaper, advertisement etc. which are digitized and also available as per user requirement. With respect to access these in terms of images from databases, different methods have been implemented to retrieve image as per usefulness. The content based image retrieval system is the application of computer vision with reference to the viewpoint of image problem. This paper is based on the automatic retrieval of most similar images to the given Query image on the basis of local feature extraction like colors, shapes, texture etc. In image retrieval system for searching, browsing, and retrieving images from a large database of images. Most usual and general method of image retrieval, make use of some method of adding up metadata such as descriptions, keywords , tokens, captioning, to the images so that retrieval can be performed well. Few systems are functioning with lower level features; manually image annotation is time-consuming, difficult and costly. To address this, many researchers proposed an automatic user friendly image retrieval system using different methods. Searching any query image which is Content-based (which might be referred to colors, shapes, textures, or any other information ) analyzed for actual image to be present in database. In this paper, we try to provide best solution in large number of data.

\section{COLOR IMAGE}

Any colored image is a blend of few basic colors. In this each individual pixel of a color image down into Red, Green and

\footnotetext{
Revised Manuscript Received on December 30, 2019.

* Correspondence Author

Bably Dolly, Ph.D., Department of Computer Science, Babasaheb Bhimrao Ambedkar University, Lucknow.

Deepa Raj, Assistant professor , Department of Computer Science Babasaheb Bhim Rao Ambedkar University

(C) The Authors. Published by Blue Eyes Intelligence Engineering and Sciences Publication (BEIESP). This is an open access article under the CC
} BY-NC-ND license (http://creativecommons.org/licenses/by-nc-nd/4.0/)
Blue values. For different color band R, G \& B different matrix is generated. The three matrices are arranged in sequential order, next to each other creating a 3 dimensional $\mathrm{m}$ by $\mathrm{n}$ of 3 matrixes. A RGB image converted to an indexed image which reduces the number of colors in the process.

\section{COLOR IMAGE FEATURE EXTRACTION}

There are different features related to the colored images:-

\subsection{Color histogram:}

An image histogram refers to the probability mass function of the image intensities. This is extensive for color images to confine the joint probability of the intensities of the three different color channels. More formally, the color histogram is defined as:

$$
\begin{aligned}
& H_{c 1, c 2, c 3}\left(x_{v} y_{v} z\right) \\
& =P_{x} \operatorname{Prob}\left(c 1=x_{v} c 2=y_{v} c 3=z\right)
\end{aligned}
$$

Where c1, c2 and c3 are the three channels of color model image (RGB, HSV, $\mathrm{L}^{*} \mathrm{a} * \mathrm{~b}$ and $\mathrm{YCbCr}$ ) and the $\mathrm{P}$ is the no of pixels in given image

\subsubsection{Color Histogram Euclidean distance:}

There are three distance formulas that can be used for image retrieval: histogram Euclidean Distance, histogram intersection and histogram quadratic (cross) distance. In this paper, histogram Euclidean Distance has been chosen to work. By considering the H' and H" as two color histogram of Query image and searched image respectively. The Euclidean Distance between the H' and H" can be computed as:

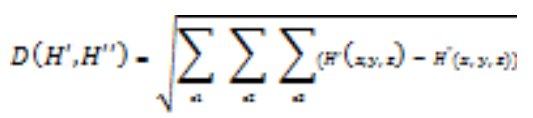

Where $\mathrm{E}$ is the Euclidean distance to be calculated between two color histogram H' and H'. In the above formula maximum of histogram value of individual channel of an image has been taken.

\subsection{Color Moments}

The second feature has been taken as color moments. The Mean and standard deviation of each channel have been calculated as:

$\operatorname{Mean}(\mathrm{M})=\frac{1}{n} \sum_{j=1}^{m} \mathrm{Ii}_{\mathrm{i}, \mathrm{j}}$

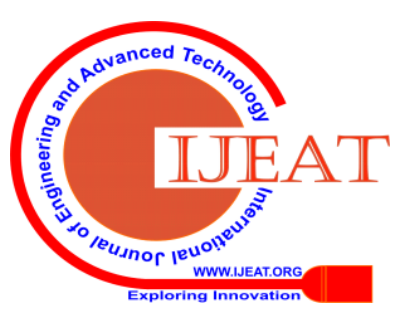


Standard Deviation $(\delta)=\sqrt{\sum_{k=0}^{n}\left(H_{i} j-E r_{i} i\right)^{k}}$

\subsubsection{Color Moments Euclidean distance:}

The Euclidean distance of mean and standard deviation respectively can be computed as:

$D_{M r}=\sqrt{\left(M^{p}-M^{p r}\right)^{2}}$

$D_{\text {nas }}=\sqrt{\left(\delta^{\prime}-\delta^{r}\right)^{2}}$

\subsection{Colormap:}

The Coplormap which gives three column matrix of RGB triplets. Each one of the row of matrix define single RGB triplet that specifies one color of the colormap. The values are in the range. There will be any length of the colormaps, but there should be widely three columns. Each one of the row in the matrix shows one color by use of an RGB triplet. There are number of elements which specify the intensities of the red, green, and blue components of the color of RGB triplets which is three-element row vector. The intensities must be in between the range. A 0 value shows no color and 1 value shows full intensity

3.3.1 Colormap Euclidean distance: The

colormap Euclidian distance can be compute as :

$\mathrm{D}_{\operatorname{mxp}}=\sqrt{\sum_{n=1}^{I}\left(c_{n}^{\prime}-c^{n}\right)^{z}}$

Where $D_{\text {map }}$ Euclidian distance for Colormap, $c_{n}$ is the number of channels i.e, where $n=1,2,3$ for three channels respectively.

\section{CBIR SYSTEM ON THE BASIS OF COLOR FEATURES}

Proposed algorithm uses combinations of color feature to overcome the problem description. The Framework of CBIR system is displayed in figure 5. Query image having similar characteristic from database has to retrieve. Proposed system is implemented and concentrated on visual contents of an image specially color applying on the roses dataset. Proposed system retrieve image from image databases with common, feature values as color histogram. In this paper, the proposed work will try to provide a platform to extract images from the database using query method. Input image before and after segmentation is shown in figure 3 .
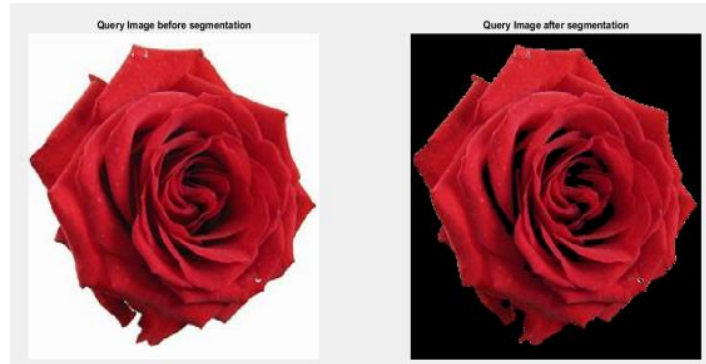

Figure3: input image (a) before segmentation (b) after segmentation

Segmented image is shown in figure 4 using different color model.

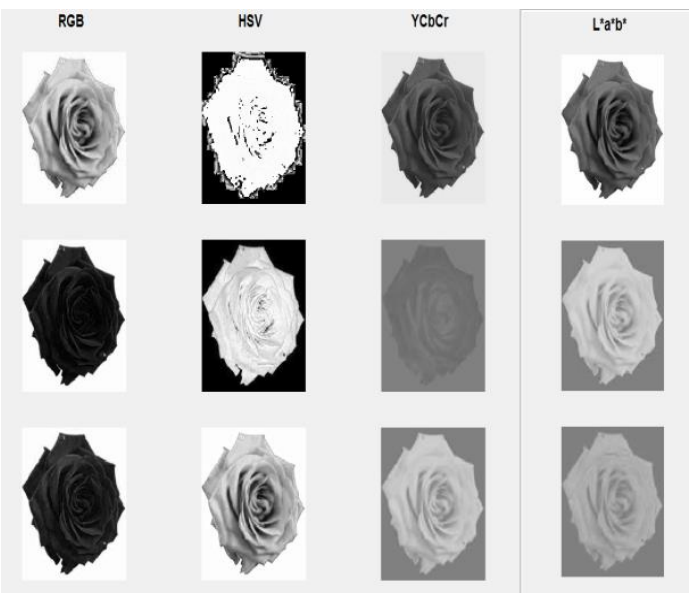

Figure4: different Color Models for input image Database of the image set are display in figure 6.

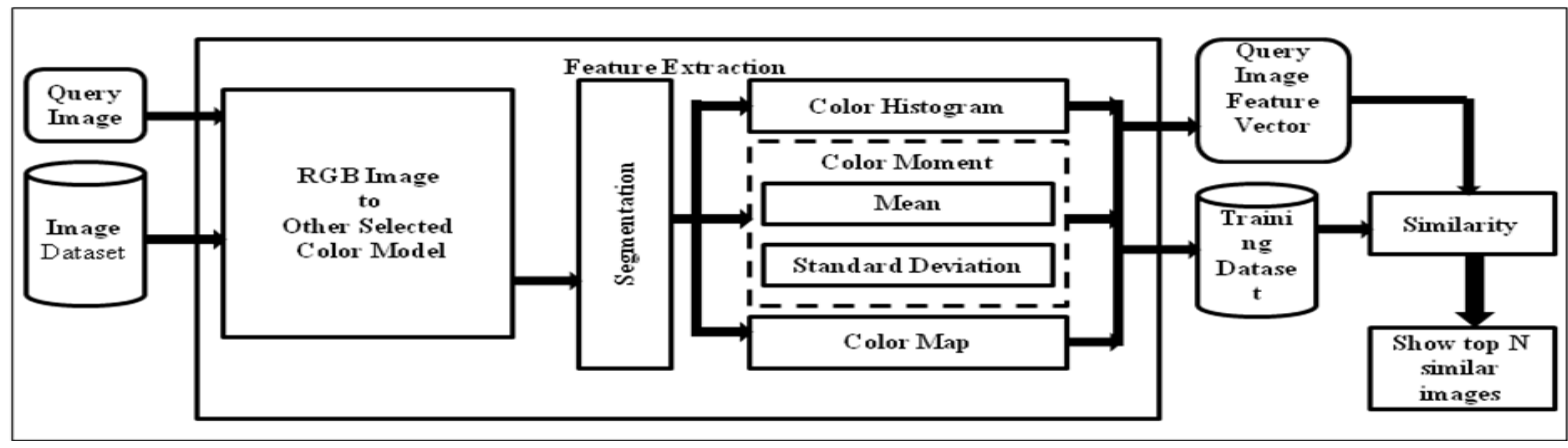

Figure 5: Framework of CBIR System 


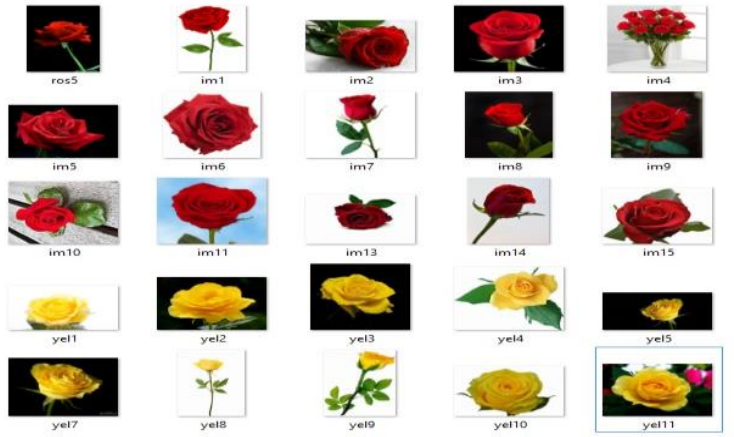

Figure 6: Set of Images in database

Following algorithm are used to retrieve the images:

1. Input a query image.

2. Convert RGB image to specified color model space.

3. Do Color Segmentation.

[Collect four features]

4. Create histogram of segmented image.

5. Take maximum of histogram value of each channel of color image separately for first feature

6. Next to take color moment: mean and standard deviation as second and third feature respectively.

7. Take color map value of each channel of segmented color image separately as fourth feature

8. Repeat from step 2 to step 7. For each image from dataset of images and store feature vector of each image.

9. Calculate feature vector of query image. Used Euclidean distance to find the score of each feature present in dataset related to query image.

10. Sort the score to retrieve top $\mathrm{N}$ similar images.

\section{EXPERIMENTAL ANALYSIS}

In this research paper different color model as RGB, YCbCr and HSV have been taken for feature collections which are as mention below:

Feature1 for RGB model: maximum value of $R, G$, and $B$ in RGB Histogram

Feature2: Mean of image intensity

Feature3: Standard Deviation of RGB image
Feature4: Color Map value of RGB

Same has been carried out for the next YCbCr, HSV and $\mathrm{L}^{*} \mathrm{a}$ b models respectively, Table1 shows the feature of query image in different models.

To retrieve the image according to the query image, score is calculated as given formula:

Distance Vector $=\sum_{1_{1}}^{n}\left(F n-F q_{n}\right)^{2}$

Where $\mathrm{n}$ denotes the number of $\mathrm{F}$ features of dataset image I, $\mathrm{q}_{\mathrm{n}}$ denotes the number of features for an query image.

After taking four features as mentioned above the Euclidian distance (D) has been calculated, Feature1 is taken as F1 for $\mathrm{D}\left(\mathrm{H}^{\prime}, \mathrm{H}^{\prime \prime}\right)$, Feature2 as F2 for $\mathrm{D}_{\mathrm{M}}$, Feature3 as F3 for $\mathrm{D}_{\text {std, }}$, Feature4 as $\mathrm{F} 4$ for $\mathrm{D}_{\text {map }}$ In this research paper, Number of features $\left(\mathrm{F}_{1 . . \mathrm{n}}\right.$ for four features of dataset images and $\mathrm{Fq}_{1 . . \mathrm{n}}$ for four features of query image) are taken, the distance vector for these four image can be calculated as:

Distance Vector $(D)=\sqrt{\left(F 1-F q_{1}\right)^{2}+\left(F 2-F q_{2}\right)^{2}+\left(F 3-F q_{3}\right)^{2}+\left(F 4-F q_{4}\right)^{2}}$

Here $\mathrm{D}$ is considering as a score card for each images in the dataset.

Where

$\mathrm{F} 1=\mathrm{R}^{\mathrm{max}}, \mathrm{G}^{\mathrm{max}}, \mathrm{B}^{\mathrm{max}}$ of Histogram of Image

F2 = Color Map value of RGB color

F3= mean of image

F4=Standard Deviation of image

Same has been taken for Query image as $\mathrm{Fq}_{1}, \mathrm{Fq}_{2}, \mathrm{Fq}_{3}, \mathrm{Fq}_{4}$ respectively.

The maximum value of color histogram of each channel in different color model for each image in database is shown in Table 2. The score of the dataset images are arranged in ascending order by using the RGB color model in Table 3 with respect to query image. Table 4 display the score of the image in YCbCr model with respect to query image. Table 5 display the score of the image in $\mathrm{L}^{*} \mathrm{a} * \mathrm{~b}$ model with respect to query image. Table 6 display the score of the image in HSV model with respect to query image. From the given tables we analyzed that YCbCr and RGB not retrieve a related image from top 1 to 14 images. $L^{*} a^{*} b$ retrieve top 14 image of same color and HSV retrieve top 14 of same color.

Table 1: features extracted for Query Image

\begin{tabular}{|c|c|c|c|c|c|c|c|c|c|c|}
\hline \multirow{3}{*}{ Sn. } & \multirow{3}{*}{$\begin{array}{l}\text { Query } \\
\text { Image }\end{array}$} & \multirow{3}{*}{$\begin{array}{l}\text { Color } \\
\text { Models }\end{array}$} & \multicolumn{3}{|c|}{$\mathrm{F} 1$} & \multicolumn{3}{|c|}{ F2 } & F3 & $\mathrm{F} 4$ \\
\hline & & & \multicolumn{3}{|c|}{ max value of Histogram } & \multicolumn{3}{|c|}{ Color Map } & \multirow{2}{*}{ Mean } & \multirow{2}{*}{$\begin{array}{l}\text { Standard } \\
\text { Deviation }\end{array}$} \\
\hline & & & Channel1 & Channel2 & Channel3 & Channel1 & Channel2 & Channel3 & & \\
\hline 1. & Red2 & $\mathrm{L}^{*} \mathrm{a} * \mathrm{~b}$ & 44005 & 48590 & 48800 & 0.1529 & 0.0117 & 0.0117 & 0.0614 & 0.1609 \\
\hline 2. & Red 2 & RGB & 40620 & 46276 & 47635 & 0.1568 & 0.0039 & 0.0039 & 14.5222 & 40.0809 \\
\hline 3. & Red 2 & HSV & 46211 & 50367 & 50427 & 0.1568 & 0.0078 & 0.0078 & 15.0010 & 42.9606 \\
\hline 4. & Red 2 & $\mathrm{YCbCr}$ & 39666 & 45014 & 45549 & 0.1921 & 0.0156 & 0.0156 & 19.4967 & 46.7806 \\
\hline
\end{tabular}

Table 2: Maximum value of color histogram of each channel separately

\begin{tabular}{|c|c|c|c|c|c|c|c|c|c|c|c|c|c|}
\hline \multirow[b]{3}{*}{ Sn. } & \multirow[b]{3}{*}{ Image } & \multicolumn{12}{|c|}{ Color Models Histogram Values } \\
\hline & & \multicolumn{3}{|c|}{$\mathbf{L} * \mathbf{a} * \mathbf{b}$} & \multicolumn{3}{|c|}{ HSV } & \multicolumn{3}{|c|}{ YCbCr } & \multicolumn{3}{|c|}{ rgb } \\
\hline & & $\max (\mathrm{L})$ & $\max (a)$ & $\max (b)$ & $\max (\mathbf{H})$ & $\max (\mathrm{S})$ & $\max (V)$ & $\max (\mathbf{Y})$ & $\max (\mathbf{C b})$ & $\max (\mathrm{Cr})$ & $\max (\mathbf{R})$ & $\max (G)$ & $\max (B)$ \\
\hline 1 & Red1 & 57423 & 60483 & 60839 & 51721 & 57722 & 57702 & 51550 & 57215 & 57591 & 51721 & 57722 & 57702 \\
\hline
\end{tabular}


Color Based Image Retrieval by Combining Various Features

\begin{tabular}{|c|c|c|c|c|c|c|c|c|c|c|c|c|c|}
\hline 2 & Red 10 & 57217 & 58462 & 57576 & 53124 & 54637 & 53421 & 55057 & 56500 & 55456 & 53124 & 54637 & 53421 \\
\hline 3 & Red 11 & 43901 & 51170 & 45199 & 41417 & 50370 & 42600 & 40447 & 49478 & 41929 & 41417 & 50370 & 42600 \\
\hline 4 & Red 12 & 57032 & 60270 & 59046 & 54544 & 57739 & 56541 & 54594 & 58530 & 57041 & 54544 & 57739 & 56541 \\
\hline 5 & Red 13 & 54305 & 57435 & 54933 & 54815 & 57456 & 55227 & 53351 & 56794 & 54192 & 54815 & 57456 & 55227 \\
\hline 6 & Red 14 & 52279 & 55319 & 53130 & 53528 & 56176 & 54326 & 51313 & 54547 & 52343 & 53528 & 56176 & 54326 \\
\hline 7 & Red 15 & 42105 & 42907 & 42702 & 44221 & 44928 & 44655 & 40400 & 41226 & 41055 & 44221 & 44928 & 44655 \\
\hline 8 & Red 2 & 44005 & 48590 & 48800 & 46211 & 50367 & 50427 & 39666 & 45014 & 45549 & 46211 & 50367 & 50427 \\
\hline 9 & Red 3 & 47467 & 52808 & 47499 & 42741 & 49851 & 42766 & 43996 & 50656 & 44044 & 42741 & 49851 & 42766 \\
\hline 10 & Red 4 & 55896 & 56663 & 56204 & 54460 & 55644 & 54692 & 54154 & 55157 & 54510 & 54460 & 55644 & 54692 \\
\hline 11 & Red 5 & 40515 & 50975 & 42149 & 41452 & 51349 & 42755 & 39925 & 50764 & 41780 & 41452 & 51349 & 42755 \\
\hline 12 & Red 7 & 59620 & 60828 & 59754 & 59125 & 60314 & 59229 & 59092 & 60344 & 59262 & 59125 & 60314 & 59229 \\
\hline 13 & Red8 & 61481 & 62681 & 62045 & 58074 & 60470 & 59423 & 59227 & 61105 & 60298 & 58074 & 60470 & 59423 \\
\hline 14 & Red 9 & 47347 & 53170 & 50212 & 44398 & 50864 & 47734 & 44258 & 51153 & 47990 & 44398 & 50864 & 47734 \\
\hline 15 & Yel1 & 65536 & 65536 & 65536 & 65536 & 65536 & 65536 & 49891 & 49891 & 53604 & 65536 & 65536 & 65536 \\
\hline 16 & Yel10 & 65342 & 65344 & 65500 & 65534 & 65536 & 65536 & 40085 & 40087 & 46795 & 65534 & 65536 & 65536 \\
\hline 17 & Yel11 & 61988 & 62917 & 62087 & 60884 & 61806 & 60885 & 37277 & 38259 & 38773 & 60884 & 61806 & 60885 \\
\hline 18 & Yel2 & 65503 & 65503 & 65513 & 65536 & 65536 & 65536 & 38848 & 38848 & 43132 & 65536 & 65536 & 65536 \\
\hline 19 & Yel3 & 65536 & 65536 & 65536 & 65536 & 65536 & 65536 & 46244 & 46244 & 49625 & 65536 & 65536 & 65536 \\
\hline 20 & Yel4 & 65527 & 65527 & 65535 & 62592 & 62592 & 62592 & 49018 & 49018 & 49439 & 62592 & 62592 & 62592 \\
\hline 21 & & 90 & 390 & 32 & 36 & 36 & 36 & & 54 & & & & 536 \\
\hline 22 & Yel7 & 65194 & 65194 & 65200 & 65536 & 65536 & 65536 & 49653 & 49653 & 50735 & 65536 & 65536 & 65536 \\
\hline 23 & & 36 & 536 & 536 & 536 & 5536 & 65536 & 2237 & 237 & 66 & & & 5536 \\
\hline 24 & Yel9 & 65536 & 65536 & 65536 & 65284 & 65278 & 65303 & 60204 & 60204 & 61245 & 65284 & 65278 & 65303 \\
\hline
\end{tabular}

\begin{tabular}{|c|c|c|c|c|c|c|}
\hline \multicolumn{7}{|c|}{ Table3: Score in RGB color model } \\
\hline & & RGB & Featc & Extr: & & \\
\hline 1 & images & F1 & F2 & $\mathbf{F 3}$ & F4 & \\
\hline$\frac{1}{2}$ & Red 11 & 6362 & 0.00 & $\frac{0.00}{6.26}$ & $\frac{0.00}{12.02}$ & 6380 \\
\hline 3 & Red 3 & 6474 & 0.03 & 3.56 & 9.65 & \\
\hline 4 & Ye15 & 7136 & 0.15 & 13.42 & 38.52 & 7188 \\
\hline 5 & Red 5 & 8683 & 0.02 & 1.26 & 2.90 & 8687 \\
\hline 6 & $\operatorname{Red} 9$ & 10929 & 0.02 & 3.02 & 3.14 & 10936 \\
\hline 7 & Ye13 & 13377 & 0.16 & 13.62 & 38.40 & 13429 \\
\hline 8 & Red 15 & 13543 & 0.07 & 5.56 & 10.60 & \\
\hline 9 & Ye12 & 15596 & 0.16 & 13.56 & 37.36 & \\
\hline 10 & Red 14 & 16202 & 0.08 & 6.87 & 14.14 & 16223 \\
\hline$\frac{11}{12}$ & Red 10 & 18023 & 0.04 & $\frac{0.17}{2.87}$ & 3.84 & $\frac{10313}{18029}$ \\
\hline 13 & Red & 18278 & 0.0 & 2.23 & 4.43 & 18285 \\
\hline 14 & Ye111 & $18 \mathrm{C}$ & 0.12 & 9.68 & 13.77 & \\
\hline & Red 13 & 18661 & 0.09 & 7.95 & 15.79 & \\
\hline 16 & Ye17 & 19633 & 0.15 & 13.27 & 38.26 & \\
\hline & & 218 & & 5.93 & 3.81 & \\
\hline 18 & Red7 & 27834 & 0.11 & 9.89 & 14.99 & \\
\hline 19 & $\mathrm{Ye} 1$ & & 0.16 & 14.48 & 39.40 & \\
\hline 20 & Yel1o & $\begin{array}{l}36114 \\
36203\end{array}$ & 0.16 & 14.51 & 39.34 & 36168 \\
\hline$\frac{21}{22}$ & $\begin{array}{l}\text { Yel4 } \\
\text { Ye11 }\end{array}$ & $\begin{array}{l}36223 \\
36224\end{array}$ & $\begin{array}{l}0.16 \\
0.16\end{array}$ & $\frac{14.32}{14.52}$ & $\begin{array}{l}40.05 \\
40.08\end{array}$ & \\
\hline 23 & Ye18 & 36224 & 0.16 & 14.52 & 40.08 & 36279 \\
\hline 24 & sed & & & 1.15 & 8.62 & 38610 \\
\hline
\end{tabular}

\begin{tabular}{|c|c|c|c|c|c|c|}
\hline \multicolumn{7}{|c|}{ Tables: Score in L*a*b color model } \\
\hline & & $\mathbf{L}^{* \mathbf{a}^{*} \mathbf{b}}$ & eature & Extra: & & \\
\hline 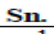 & images & F1 & F2 & $\mathbf{F 3}$ & F4 & Scon \\
\hline & $\operatorname{Red} 2$ & & 0.00 & 0.00 & 0.00 & \\
\hline$\frac{2}{3}$ & ed11 & 4431 & 0.04 & 0.01 & 0.03 & 4431 \\
\hline$\frac{3}{4}$ & $\frac{e d 3}{20}$ & $\frac{5610}{5843}$ & & 0.01 & $\frac{0.03}{0.01}$ & $\frac{5610}{5843}$ \\
\hline 4 & Non & 7881 & & & & 7881 \\
\hline 6 & Red15 & 8549 & 0.05 & 0.04 & $\frac{0.03}{0.03}$ & 8549 \\
\hline & Red14 & 11510 & 0.07 & 003 & & 11510 \\
\hline 8 & Red13 & 14898 & 0.08 & 0.03 & 0.06 & 14898 \\
\hline 9 & Red4 & 16168 & 0.06 & 0.02 & 0.01 & 16168 \\
\hline 10 & Red10 & 18682 & 0.06 & 0.02 & 0.01 & 18682 \\
\hline 11 & Red12 & 20276 & 0.10 & 0.04 & 0.07 & 20276 \\
\hline 12 & $\operatorname{Rec}$ & 213 & 0.09 & 0.04 & 0.05 & \\
\hline 13 & Red7 & 22662 & 0.10 & 0.04 & 0.05 & 22663 \\
\hline 14 & Red8 & & 0.12 & 0.05 & 0.08 & 26065 \\
\hline 15 & Yel11 & 26556 & 0.12 & 0.05 & 0.07 & 26556 \\
\hline 16 & $\begin{array}{l}\text { Ye17 } \\
Y e 11\end{array}$ & $\frac{31522}{31857}$ & 0.15 & 0.06 & 0.13 & $\frac{31522}{31857}$ \\
\hline 18 & Yel15 & 31857 & $\begin{array}{l}0.13 \\
0.15\end{array}$ & 0.06 & $\frac{0.14}{0.14}$ & $\begin{array}{l}31857 \\
31878\end{array}$ \\
\hline 19 & Ye12 & 32055 & 0.15 & 0.06 & 0.15 & 32056 \\
\hline 20 & Yel4 & 32095 & 0.15 & 0.06 & 0.16 & \\
\hline 21 & Yel & 32107 & 0.15 & 0.06 & 0.16 & 32107 \\
\hline 22 & Ye13 & 32107 & 0.15 & 0.06 & 0.16 & \\
\hline 23 & Ye18 & 32107 & 0.15 & 0.06 & 0.16 & 2107 \\
\hline 24 & Ye19 & 32107 & & 0.06 & 0.16 & 32107 \\
\hline
\end{tabular}

\begin{tabular}{|c|c|c|c|c|c|c|}
\hline \multirow[b]{2}{*}{ Sn. } & \multirow[b]{2}{*}{ images } & \multicolumn{4}{|c|}{ YCbCr F eature Extracted } & \multirow[b]{2}{*}{ Score } \\
\hline & & F1 & F2 & F3 & F4 & \\
\hline & Red2 & 0 & 0.00 & 0.00 & 0.00 & o \\
\hline 2 & Yel10 & 5099 & 0.22 & 24.29 & 26.66 & 5151 \\
\hline 3 & Red11 & 5800 & 0.04 & 2.14 & 6.02 & 5808 \\
\hline 4 & Red15 & 5923 & 0.03 & 4.14 & 2.58 & 5930 \\
\hline 5 & Yel2 & 6673 & 0.30 & 35.20 & 42.45 & 6751 \\
\hline 6 & $\operatorname{Red} 5$ & 6880 & 0.05 & 5.37 & 8.16 & 6894 \\
\hline 7 & $\operatorname{Red} 3$ & 7270 & 0.02 & 2.52 & 7.40 & 7279 \\
\hline 8 & Ye13 & 7836 & 0.17 & 16.88 & 26.87 & 7880 \\
\hline 9 & $\operatorname{Red} 9$ & 8046 & 0.03 & 3.19 & 2.59 & 8051 \\
\hline 10 & Yel11 & 9862 & 0.25 & 31.66 & 35.27 & 9929 \\
\hline 11 & Yel4 & 10891 & 0.17 & 19.83 & 31.56 & 10943 \\
\hline 12 & Yel7 & 12172 & 0.13 & 10.21 & 20.10 & 12202 \\
\hline 13 & Yel1 & 13900 & 0.18 & 19.85 & 37.53 & 13958 \\
\hline 14 & Red 14 & 16513 & 0.11 & 10.17 & 17.38 & 16541 \\
\hline 15 & Red4 & 19826 & 0.07 & 6.68 & 3.72 & 19837 \\
\hline 16 & Red 13 & 20019 & 0.12 & 11.39 & 19.31 & 20050 \\
\hline 17 & Red 1 & 20859 & 0.06 & 7.09 & 2.66 & 20869 \\
\hline & Red 10 & 21609 & & 6.92 & & 21618 \\
\hline 19 & Red 12 & 23186 & 0.12 & 12.21 & 15.54 & 23214 \\
\hline 20 & Ye15 & 24470 & 0.11 & 3.40 & 4.06 & 24477 \\
\hline 21 & Red7 & 28292 & 0.14 & 13.05 & 16.96 & 28322 \\
\hline 22 & $\operatorname{Red} 8$ & 29310 & 0.14 & 13.86 & 17.00 & 29341 \\
\hline 23 & Ye19 & 29982 & 0.12 & 7.85 & 1.07 & 29991 \\
\hline 24 & Yel8 & 32948 & 0.15 & 10.78 & 5.09 & 32964 \\
\hline
\end{tabular}

\begin{tabular}{|c|c|c|c|c|c|c|}
\hline \multicolumn{7}{|c|}{ Table6: Score in HSV color model } \\
\hline & & HSV F & eature & Etract & & \\
\hline Sn. & images & F1 & F2 & F3 & F4 & Score \\
\hline & Red2 & 0 & & 0.00 & 0.00 & \\
\hline$\frac{1}{2}$ & Red9 & 3284 & 0.01 & 1.92 & & 3288 \\
\hline$\frac{2}{3}$ & Red15 & 8177 & 0.04 & 5.66 & 4.61 & 8187 \\
\hline$\frac{3}{4}$ & Red3 & 8426 & 0.08 & 9.23 & 14.94 & 8450 \\
\hline 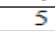 & Red10 & 8659 & 0.01 & 0.35 & 7.84 & 8668 \\
\hline 6 & Red5 & 9081 & 0.08 & 9.15 & 11.59 & 9102 \\
\hline & Red1 1 & 9178 & 0.07 & 5.96 & 9.48 & \\
\hline 8 & Red14 & 10123 & 0.08 & 7.22 & 16.20 & 10147 \\
\hline 9 & Red4 & 10681 & 0.04 & 2.46 & 0.95 & 10684 \\
\hline 10 & Red1 & 11721 & 0.02 & 2.63 & 1.90 & 11726 \\
\hline 11 & Red13 & 12138 & 0.09 & 7.53 & 16.48 & 12162 \\
\hline 12 & Red12 & 12695 & 0.06 & 6.03 & 5.99 & 12707 \\
\hline 13 & Red8 & 17992 & 0.08 & 7.81 & 7.10 & 18007 \\
\hline 14 & Red7 & 18525 & 0.10 & 8.86 & 14.10 & 18548 \\
\hline 15 & Yel11 & 21343 & 0.11 & 8.56 & 11.04 & 21363 \\
\hline 16 & Yel4 & 23786 & 0.15 & 12.19 & 27.96 & 23826 \\
\hline 17 & Ye19 & 28415 & 0.16 & 14.85 & 39.82 & 28470 \\
\hline 18 & Yel10 & 28840 & 0.16 & 15.00 & 42.71 & 28898 \\
\hline 19 & Yel1 & 28842 & 0.16 & 15. & 42.96 & \\
\hline & $\mathrm{Ye}$ & 18842 & 0.16 & 15. & 42.96 & \\
\hline 21 & Ye13 & 28842 & 0.16 & 15 & 42.96 & \\
\hline 22 & Ye15 & 28842 & 0.16 & 15.00 & 42.96 & 28900 \\
\hline 23 & Ye17 & 28842 & 0.16 & 15.00 & 42.96 & 28900 \\
\hline 24 & Ye18 & 28842 & 0.16 & 15.00 & 42.96 & 28900 \\
\hline
\end{tabular}


Table 7 and Table 8 use to compare the retrieval rate of HSV and $\mathrm{L}^{*} \mathrm{a} * \mathrm{~b}$ for best result. Three categories of databases of specific color images have been taken like apple dataset, bus dataset, flower dataset to compare with the proposed method as well as famous precision and recall measures have been calculated for given pre-categorized databases. Both precision and recall are strong evaluation measure. Most of each experiment, image retrieval performed for given every query image. In this regard all the red images in different dataset are renamed as ' $r$ ', green as ' $g$ ' and yellow as ' $y$ ' in table 7 and table 8 . In table7 using HSV model, it retrieved all top similar red images from apple dataset, and same retrieve all top in bus dataset with reference to query image and retrieved all top red images from flower dataset. $\mathrm{L}^{*} \mathrm{a} * \mathrm{~b}$ color model it also gives the same result for apple dataset and bus dataset and lower dataset.

Table7: HSV color image score for three different categorized dataset

\begin{tabular}{|c|c|c|c|c|c|}
\hline \multicolumn{6}{|c|}{ HSV color image score for three different dataset } \\
\hline apple & score & bus & score & flower & score \\
\hline red1 & 0 & r2 & 0 & r2 & 0 \\
\hline r13 & 1293 & r12 & 1267 & r9 & 3288 \\
\hline r15 & 2564 & r5 & 1992 & r15 & 8187 \\
\hline r12 & 4132 & r7 & 3483 & r3 & 8450 \\
\hline r9 & 4233 & r17 & 3773 & r10 & 8668 \\
\hline r8 & 5707 & r3 & 5053 & r5 & 9102 \\
\hline r7 & 6416 & r6 & 5582 & r11 & 9194 \\
\hline r14 & 9033 & r4 & 5755 & r14 & 10147 \\
\hline r11 & 9054 & r14 & 6124 & r4 & 10684 \\
\hline r6 & 15667 & r8 & 8052 & r1 & 11726 \\
\hline r2 & 16902 & r15 & 8083 & r13 & 12162 \\
\hline r3 & 16902 & r16 & 8087 & r12 & 12707 \\
\hline r4 & 17839 & r10 & 13043 & r8 & 18007 \\
\hline r10 & 18558 & r11 & 14442 & r7 & 18548 \\
\hline r5 & 19621 & r9 & 14816 & y11 & 21363 \\
\hline g16 & 24171 & r1 & 21162 & y4 & 23826 \\
\hline g17 & 28078 & y4 & 24172 & y9 & 28470 \\
\hline g18 & 28078 & y3 & 24889 & y10 & 28898 \\
\hline g19 & 28078 & y6 & 25090 & y1 & 28900 \\
\hline g20 & 28078 & y7 & 25190 & y2 & 28900 \\
\hline g21 & 28078 & y9 & 25199 & y3 & 28900 \\
\hline g22 & 28078 & y2 & 25220 & y5 & 28900 \\
\hline y23 & 28078 & y5 & 25221 & y7 & 28900 \\
\hline y24 & 28078 & y1 & 25223 & y8 & 28900 \\
\hline y25 & 28078 & y8 & 25223 & r6 & 31884 \\
\hline
\end{tabular}

Table 8: $\mathbf{L}^{*} \mathbf{a} * \mathbf{b}$ color image score for three different categorized dataset

\begin{tabular}{|c|c|c|c|c|c|}
\hline \multicolumn{6}{|c|}{ L*a*b color image score for different category of dataset } \\
\hline apple & score & bus & score & flower & score \\
\hline r1 & 0 & r2 & 0 & r2 & 0 \\
\hline r2 & 2844 & r5 & 707 & r11 & 4431 \\
\hline r6 & 3977 & $\mathrm{r} 14$ & 1046 & r3 & 5610 \\
\hline r5 & 4622 & r8 & 1061 & r9 & 5843 \\
\hline r11 & 7070 & r15 & 3130 & r5 & 7881 \\
\hline r8 & 1163 & r12 & 5946 & r15 & 8549 \\
\hline r13 & 1331 & r10 & 6936 & r14 & 11510 \\
\hline
\end{tabular}

\begin{tabular}{|c|c|c|c|c|c|} 
r15 & 1359 &.$r 4$. & 7831 & r13 & 14898 \\
\hline r12 & 1699 & r7 & 9647 & r4 & 16168 \\
\hline r9 & 1882 & r3 & 10375 & r10 & 18682 \\
\hline r2 & 2138 & r17 & 10828 & r12 & 20276 \\
\hline r3 & 2131 & r1 & 12287 & r1 & 21597 \\
\hline r7 & 2266 & r6 & 13385 & r7 & 22663 \\
\hline r4 & 2389 & r16 & 15754 & r8 & 26065 \\
\hline r14 & 4418 & r11 & 19363 & y11 & 26556 \\
\hline y24 & 4903 & r9 & 21251 & r6 & 30840 \\
\hline g16 & 4917 & y6 & 34238 & y7 & 31522 \\
\hline g17 & 4919 & y3 & 34290 & y10 & 31857 \\
\hline g18 & 4919 & y9 & 34458 & y5 & 31878 \\
\hline g19 & 4919 & y4 & 34597 & y2 & 32056 \\
\hline g20 & 4919 & y2 & 34721 & y4 & 32096 \\
\hline g21 & 4919 & y1 & 34785 & y1 & 32107 \\
\hline g22 & 4919 & y7 & 34817 & y3 & 32107 \\
\hline y23 & 4919 & y5 & 34822 & y8 & 32107 \\
\hline y25 & 4919 & y8 & 34822 & y9 & 32107 \\
\hline
\end{tabular}

\subsection{The image retrieval Efficiency:}

The precision of the system significantly shows the total number of similar images present in retrieved images and the total number of retrieved images from the database. In the same way recall is the ratio of the number of similar images present in the retrieved images and the total number of relevant images in the database. In this paper, for given Query image Q, if total $\mathrm{N}$ numbers of images have been retrieved so for this, The retrieval efficiency named as precision, recall and accuracy can be calculated given below:

\section{Precision $=\frac{\text { Number of relevant images retrieved }}{\text { Total number of images in database }}$$$
\text { Recall }=\frac{\text { Number of relevant images retrieved }}{\text { Total no of relevant images in database }}
$$

Table9 : image retrieval results with respect to precision and recall

\begin{tabular}{|l|l|l|l|}
\hline $\begin{array}{l}\text { Proposed Techniques using } \\
\text { given HSV in different } \\
\text { image categories }\end{array}$ & $\begin{array}{l}\text { Apple } \\
\text { database }\end{array}$ & $\begin{array}{l}\text { Bus } \\
\text { database }\end{array}$ & $\begin{array}{l}\text { Flowers } \\
\text { database }\end{array}$ \\
\hline Query Image(n1) & R1 & R2 & R2 \\
\hline $\begin{array}{l}\text { Number of images in } \\
\text { database(n2) }\end{array}$ & 24 & 24 & 24 \\
\hline Recall & 1.00 & 1.00 & 0.93 \\
\hline Precision & 0.60 & 0.64 & 0.56 \\
\hline
\end{tabular}

As it can be seen from the above Table 9 the proposed technique is implemented in different category of dataset. First dataset is for collection of images of apple dataset of Red, Green and Yellow colored images, second dataset is the collection of buses of red and yellow color and the third dataset is the collection of roses of red and yellow colors.

5.2 Comparative Study by using different color models in proposed work:

Table10: Comparative Study by using different color models in proposed work

\begin{tabular}{|l|l|l|l|l|}
\hline $\begin{array}{l}\text { Proposed } \\
\text { Techniques using } \\
\text { given color model }\end{array}$ & RGB & YCbCr & L*a*b & HSV \\
\hline Input Images & Red2 & Red2 & Red2 & Red2 \\
\hline
\end{tabular}




\begin{tabular}{|l|l|l|l|l|}
\hline Precision & 0.28 & 0.25 & 0.40 & 0.40 \\
\hline Recall & 0.47 & 0.40 & 0.67 & 0.67 \\
\hline
\end{tabular}

On the basis of above comparative study of different color models by implementation of the proposed steps of CBIR system, it's clear from the above Table 10 that both $\mathrm{L}^{*} \mathrm{a} * \mathrm{~b}$ and HSV give the better result as the accuracy of both $\mathrm{L}^{*} \mathrm{a} * \mathrm{~b}$ and $\mathrm{HSV}$ are same. Both are giving the best top 14 images related to query image out of 15 relevant images. So in this paper it can be say that on the basis of color feature both HSV and $\mathrm{L}^{*} \mathrm{a}$ *b doing well their job rather than RGB and YCbCr. Since HSV and L*a*b both shows same accuracy on the basis of color features, hence in this research paper , there are mix of all three dataset(shown in figure 7) category and making the one dataset for more accurate result which is shown through the table11.

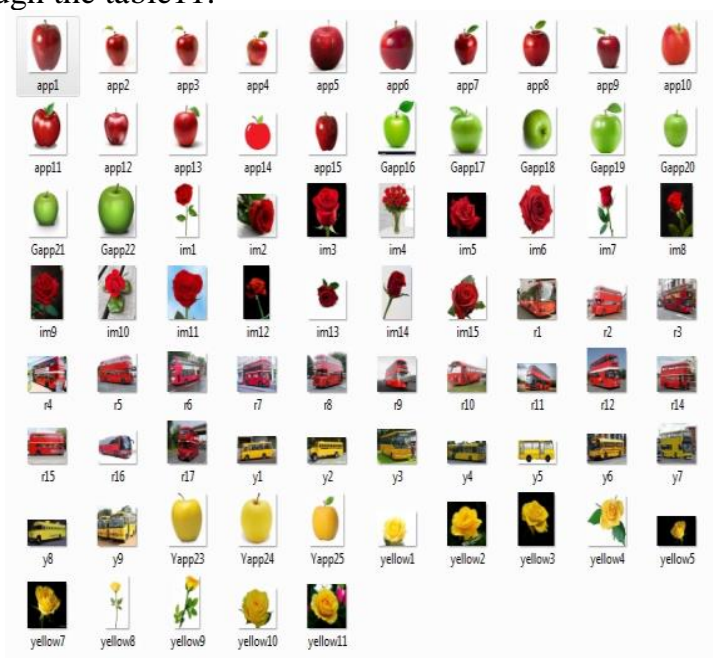

Figure 7: mixed dataset of dataset for apple, bus and flower

Table11: Comparative analysis of proposed work using HSV and $L^{*} \mathbf{a} * \mathbf{b}$ color models

\begin{tabular}{|c|c|c|c|c|}
\hline \multirow[b]{2}{*}{ sn. } & \multicolumn{2}{|c|}{ HSV } & \multicolumn{2}{|c|}{$\mathbf{L}^{*} \mathbf{a} * \mathbf{b}$} \\
\hline & image & score & image & score \\
\hline 1 & RedRose2 & 0 & RedRose2 & 0 \\
\hline 2 & RedRose9 & 3288 & RedBus15 & 3753 \\
\hline 3 & RedApple13 & 3329 & RedApple12 & 3828 \\
\hline 4 & RedApple1 & 3478 & RedApple9 & 4116 \\
\hline 5 & RedBus5 & 3728 & RedBus8 & 4199 \\
\hline 6 & RedApple15 & 3890 & RedRose11 & 4431 \\
\hline 7 & RedBus12 & 4068 & RedBus2 & 4749 \\
\hline 8 & RedBus14 & 4222 & RedBus12 & 4801 \\
\hline 9 & RedBus2 & 4854 & RedBus5 & 5255 \\
\hline 10 & RedBus15 & 5726 & RedApple2 & 5302 \\
\hline 11 & RedApple12 & 5849 & RedApple3 & 5302 \\
\hline 12 & RedApple9 & 5936 & RedApple15 & 5305 \\
\hline 13 & RedApple8 & 6093 & RedBus14 & 5404 \\
\hline 14 & RedApple7 & 7472 & RedApple13 & 5429 \\
\hline 15 & RedBus7 & 7635 & RedBus10 & 5508 \\
\hline 16 & RedBus17 & 7799 & RedBus4 & 5560 \\
\hline 17 & RedRose15 & 8187 & RedRose3 & 5610 \\
\hline 18 & RedRose3 & 8450 & RedRose9 & 5843 \\
\hline 19 & RedRose10 & 8668 & RedApple7 & 6160 \\
\hline 20 & RedApple11 & 9065 & RedApple8 & 6819 \\
\hline 21 & RedApple14 & 9085 & RedApple4 & 7566 \\
\hline 22 & RedRose5 & 9102 & RedBus7 & 7610 \\
\hline 23 & RedBus3 & 9165 & RedRose5 & 7881 \\
\hline
\end{tabular}

Published By:

Blue Eyes Intelligence Engineering 459 \& Sciences Publication

\begin{tabular}{|c|c|c|c|c|}
\hline 24 & RedRose11 & 9194 & RedBus3 & 8326 \\
\hline 25 & RedBus4 & 9481 & RedRose15 & 8549 \\
\hline 26 & RedBus6 & 9695 & RedBus17 & 8560 \\
\hline 27 & RedRose14 & 10147 & RedBus1 & 10058 \\
\hline 28 & RedRose4 & 10684 & RedApple11 & 10892 \\
\hline 29 & RedRose1 & 11726 & RedBus6 & 11111 \\
\hline 30 & RedBus8 & 12008 & RedRose14 & 11510 \\
\hline 31 & RedBus16 & 12054 & RedBus16 & 13368 \\
\hline 32 & RedRose13 & 12162 & RedRose13 & 14898 \\
\hline 33 & RedRose12 & 12707 & RedApple10 & 14973 \\
\hline 34 & RedApple6 & 15377 & RedRose4 & 16168 \\
\hline 35 & RedBus10 & 16865 & RedBus11 & 16835 \\
\hline 36 & RedApple2 & 17774 & RedApple1 & 17737 \\
\hline 37 & RedApple3 & 17774 & RedRose10 & 18682 \\
\hline 38 & RedRose8 & 18007 & RedBus9 & 18688 \\
\hline 39 & RedBus11 & 18227 & RedRose12 & 20276 \\
\hline 40 & RedBus9 & 18546 & RedApple6 & 21566 \\
\hline 41 & RedRose7 & 18548 & RedRose1 & 21597 \\
\hline 42 & RedApple4 & 18754 & RedApple5 & 22271 \\
\hline 43 & RedApple5 & 19308 & RedRose7 & 22663 \\
\hline 44 & RedApple10 & 19478 & RedRose8 & 26065 \\
\hline 45 & YellowRose11 & 21363 & YellowRose11 & 26556 \\
\hline 46 & YellowRose4 & 23826 & RedApple14 & 27142 \\
\hline 47 & RedBus1 & 24862 & YellowRose7 & 31522 \\
\hline 48 & GreenApple16 & 25016 & YellowBus6 & 31523 \\
\hline 49 & YellowBus4 & 27856 & YellowBus3 & 31579 \\
\hline 50 & YellowRose9 & 28470 & YellowBus9 & 31743 \\
\hline 51 & YellowBus3 & 28568 & YellowRose10 & 31857 \\
\hline 52 & YellowBus6 & 28767 & YellowRose5 & 31878 \\
\hline 53 & YellowBus7 & 28866 & YellowBus4 & 31884 \\
\hline 54 & YellowBus9 & 28875 & YellowApple24 & 31941 \\
\hline 55 & YellowBus2 & 28896 & YellowBus2 & 32006 \\
\hline 56 & YellowBus5 & 28898 & YellowRose2 & 32056 \\
\hline 57 & YellowRose10 & 28898 & YellowBus1 & 32070 \\
\hline 58 & GreenApple17 & 28900 & GreenApple16 & 32090 \\
\hline 59 & GreenApple18 & 28900 & YellowRose4 & 32096 \\
\hline 60 & GreenApple19 & 28900 & YellowBus7 & 32102 \\
\hline 61 & GreenApple20 & 28900 & GreenApple17 & 32107 \\
\hline 62 & GreenApple21 & 28900 & GreenApple18 & 32107 \\
\hline 63 & GreenApple22 & 28900 & GreenApple19 & 32107 \\
\hline 64 & YellowApple23 & 28900 & GreenApple20 & 32107 \\
\hline 65 & YellowApple24 & 28900 & GreenApple21 & 32107 \\
\hline 66 & YellowApple25 & 28900 & GreenApple22 & 32107 \\
\hline 67 & YellowBus1 & 28900 & YellowApple23 & 32107 \\
\hline 68 & YellowBus8 & 28900 & YellowApple25 & 32107 \\
\hline 69 & YellowRose1 & 28900 & YellowBus5 & 32107 \\
\hline 70 & YellowRose2 & 28900 & YellowBus8 & 32107 \\
\hline 71 & YellowRose3 & 28900 & YellowRose1 & 32107 \\
\hline 72 & YellowRose5 & 28900 & YellowRose3 & 32107 \\
\hline 73 & YellowRose7 & 28900 & YellowRose8 & 32107 \\
\hline 74 & YellowRose8 & 28900 & YellowRose9 & 32107 \\
\hline
\end{tabular}




\section{Color Based Image Retrieval by Combining Various Features}

\section{CONCLUSION}

Most Content-Based Image Retrieval (CBIR) systems focus on different stock of photo collections and try to address challenges of large specialized image collections and topics such as efficient image retrieval by image content. The research work concentrated on large set of images with different categories. This paper analyzed that the retrieval rate of HSV model and $\mathrm{L}^{*} \mathrm{a} * \mathrm{~b}$ model for color based image retrieval are approximately same using the proposed approach. While of RGB and YCbCr color model.

\section{REFERENCES:}

1. Xiang-Yang Wang et.al, "An effective image retrieval scheme using color, texture and shape features", Computer Standards \& Interfaces 33 (2011) page no 59-68

2. Jun You et. El, "Content-based image retrieval using color and texture fused feature" Mathematical and Computer Modeling, 54(2011), 1121-1127

3. Yogita Mistry et-al, "Content based image retrieval using hybrid features and various distance metric", Journal of Electrical Systems and Information Technology, 2017https://electronicsforu.com/electronics-rojects/prototypes/histogr am-extraction-color-image-matlab

4. Alsmadi M, Omar K. Fish, "classification: fish classification using memetic algorithms with back propagation classifier", 2012

5. Alsmadi MK, Omar KB, Noah SA.,"Fish classification based on robust features extraction from color signature using back-propagation classifier", J Comput Sci 2011, 7(1):52

6. Hany Fathy Atlam, et.al, "Comparative Study on CBIR based on Color Feature" International Journal of Computer Applications Volume 78 No.16, September 2013, ISSN 0975 - 8887

7. Mark Nixon \& Alberto Aquado, "Feature Extraction and Image Processing", second Edition 2008, Academic Press is an imprint of Elsevier, ISBN: 978-0-12372-538-7

8. R.Venkata Ramana Chary, et. al "FEATURE EXTRACTION METHODS FOR COLOR"'

9. Series Editor W. Bruce Croft, "Chapter 3: Color Feature Extraction”, The Kluwer International Series On Information Retrieval, Amherst, Kluwer Academic Publishers Massachusetts, ISBN: 0-792-37944-6

10. Mohammed Hamzah Abed, et.al, "Content based Image Retrieval based on Histogram", International Journal of Computer Applications ,Volume 110 - No. 3, January 2015 42,ISSN 0975 - 8887

\section{AUTHORS PROFILE}

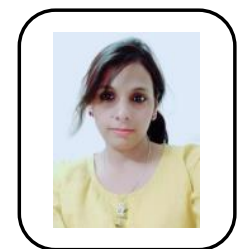

Bably Dolly pursed M.tech(Computer Science \& Technology) from Integral University Lucknow in year 2016 . She is currently pursuing Ph.D. in Department of Computer Science, Babasaheb Bhimrao Ambedkar University, Lucknow. Her main research work focuses on Digital Image Processing, Computer Vision

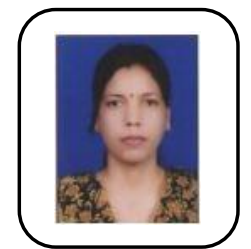

Deepa Raj, Working as an assistant professor in the Department of Computer Science Babasaheb Bhim Rao Ambedkar University. She did her Post Graduation from J.K Institute of applied physics and technology, Allahabad University and Ph.D. from Babasaheb Bhim Rao Ambedkar University Lucknow in the field of software engineering. Her field of interest is Software Engineering, Computer Graphics, and Image processing. She has attended lots of National and International conference and numbers of research papers published in her field. 